\title{
Self-Collimated beams in 2D complex periodic lattices from P- to PT-symmetry
}

\author{
$\underline{\text { W. W. Ahmed }}^{1}$, M. Botey ${ }^{1}$, R. Herrero ${ }^{1}$, K. Staliunas ${ }^{1,2}$ \\ ${ }^{1}$ Universitat Politècnica de Catalunya (UPC), Departament de Física, Colom 11, E-08222, Terrassa, Spian \\ ${ }^{2}$ Institució Catalana de Recerca i Estudis Avançats (ICREA), t, Street, Postcode, City, Country \\ waqas.waseem291@gmail.com
}

\begin{abstract}
We analyze self-collimation in two-dimensional periodic complex lattices. We consider P-symmetric and PT-symmetric complex lattices with different geometries, where the periodic modulations of both refractive index and gain/loss are either in-phase, or dephased a quarter of wavelength of the modulation. The non-diffractive propagation of light beams is analytically predicted using coupled mode approach and further confirmed by numerical integration of a paraxial model.
\end{abstract}

\section{INTRODUCTION}

Optical diffraction is a fundamental phenomenon that leads to beam broadening due to the different phase accumulation at different spatial components. Controlling diffraction may become crucial to design all optical systems. Artificial structured materials hold the ability to suppress diffraction, leading to the so-called selfcollimation phenomena (an analog of quantum mechanical dynamical localization, occurring in modulated lattices). Self-collimation was first predicted in Photonic Crystals (PhCs) [1-3] where the effect, also known as self-guiding, is accounted by flat segments in the dispersion curves of propagating modes which allow narrow beams to propagate without any distortion. It was later found that Gain and Loss Materials (GLM) modulated in the wavelength scale also hold this same property [4,5]. However, in both cases, either due to the unavoidable presence of losses in PhCs or to the anisotropy of gain in GLM, the absence of diffraction is generally accompanied by diffusive broadening. Therefore, only materials where refractive index and gain/loss are independently modulated may be expected to be able to control both diffraction and diffusion broadening. Recently, PT-symmetric complex lattices, have attracted a significant attention due to novel properties such as, unidirectional invisibility, double refraction, power oscillation [6-8]. Nevertheless, to date, complex materials are studied to control the dispersive properties for beam shaping mechanism by independently modulating refractive index and gain/loss.

In the present work, we demonstrate self-collimation in 2-dimensional (2D) Parity (P-) symmetric and Parity Time (PT) symmetric complex lattices (where index and gain/loss modulations are either in phase or de-phased a quarter of the period of the modulation) $[9,10]$. In this later case, we analyze PT-symmetry in transverse, longitudinal and simultaneously in both directions to study the mode dynamics of self-collimated beams.

\section{RESULTS AND DISCUSSION}

We study two-dimensional (2D) complex modulated potentials in the general form: $U(x, z)=4\left[m_{\mathrm{Re}} \cos (x)+i m_{\mathrm{Im}} \cos (x-\varphi)\right] \cos (Q z)$ where $m_{R e}$ and $m_{\mathrm{Im}}$ are, respectively, the normalized amplitudes of refractive index modulation and gain-loss modulation, and $Q$ is a dimensionless geometrical parameter of the lattice [6]. The phase, $\varphi$, determines the symmetry character of such a potential in the transverse direction, ranging from Psymmetry, $U(x)=U(-x)$, for $\varphi=0$, to PT-symmetry, $U(x)=U^{*}(-x)$, for $\varphi=\pi / 2$. In such complex lattices, index and gain/loss modulations allow controlling the dispersive properties of beam propagating. The specific characteristics required for the suppression of diffraction, geometries and relative amplitudes of the gain/loss and index modulations, may be analytically explored by a coupled mode analysis. The flattening features in the curvature of both, real and imaginary components of the dispersion determine the non-diffractive and nondiffusive beam propagation. Some examples of self-collimation are provided in Fig.1, indicating the 
corresponding specific 2D complex lattices (first, second and third columns of Fig.1), analytic flat dispersion bands (real dispersion is provided in the fourth column of Fig.1) and numerical propagation of Gaussian beams within the crystals (fifth column of Fig.1). Numerical validations of the predicted effect are performed in a paraxial approximation for the slowly varying amplitude of the complex field.

For the case of P-symmetric complex crystals, $\varphi=0$, beams can propagate without any broadening while being amplified due to anisotropic gain in GLM materials [5], see Fig. 1a (where the amplitude of the beam is amplified, is here depicted renormalized at each propagation step). The evolution of a beam through large propagation length, on the order several Rayleigh lengths, clearly indicates that the beam maintains its width and shape, showing the self-collimation phenomena. Exploring the modulation parameter space $\left(m_{R \mathrm{e}}, m_{I m}, Q\right)$, different regimes of simultaneous suppression of diffusion and diffraction are found, see Fig. 1a for $\varphi=0$ and Fig. $1 \mathrm{~b}$ for $\varphi=\pi / 4$. Self-collimation is also found either for collinear and non-collinear propagation in such complex crystals for phases between $\varphi=0$ and $\varphi=\pi / 2$.

As phase ranges from $\varphi=0$ and $\varphi=\pi / 2$ the mode coupling evolves from being always perfectly symmetric to supporting asymmetric coupling depending on parameters (as indicated in the inset on third column of Fig.1). PT-symmetric lattices also offer the possibility to suppress diffraction, see Fig.1c for collinear self-collimation for a PT-symmetric lattice. We show that PT-symmetric lattices may be regarded as belonging to the two limiting cases of PhC-like and GLM-like lattices, at each side of the PT-transition or exceptional point corresponding to $m_{R e}=m_{I m}$ and $\varphi=\pi / 2$. We analytically analyze the characteristics of the spatial modulations required for the suppression of diffraction, for situations ranging from the pure $\mathrm{PhC}$ limit (system displaying real eigenvalues as for the case depicted in Figs. 1c) to the pure GLM limit, that is to say, at either side of the PT-transition exceptional point. Non-diffractive beams in GLM-like systems always experience self-broadening due to intrinsic diffusion. Besides, predicting self-collimation PT-symmetry in the transverse direction (Fig. 1c), we also analyze lattices where PT-symmetry occurs in the longitudinal directional. In this last case, the situation turns out to be almost equivalent to the transverse PT-symmetric one, except for the symmetric /asymmetric energy distribution among modes. In all cases the analytical predictions are perfectly reproduced by the numerical simulations (see the last column of Fig. 1).

(a) $\varphi=0$

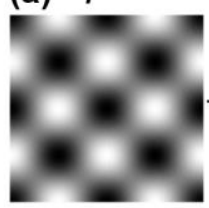

(b) $\varphi=\pi / 4$

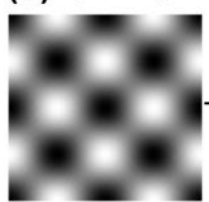

(c) $\varphi=\pi / 2$

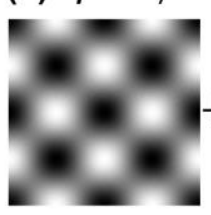

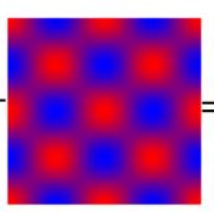
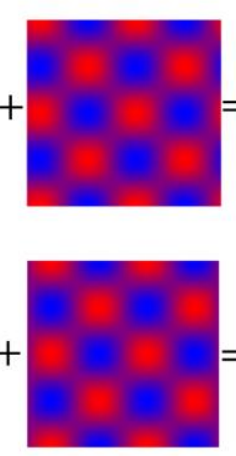
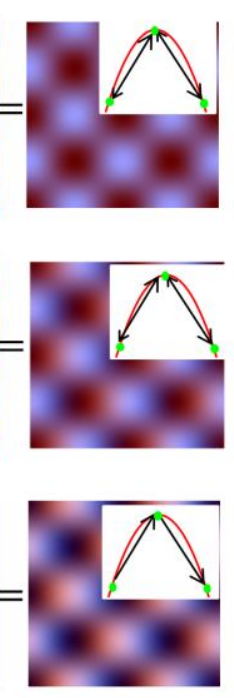
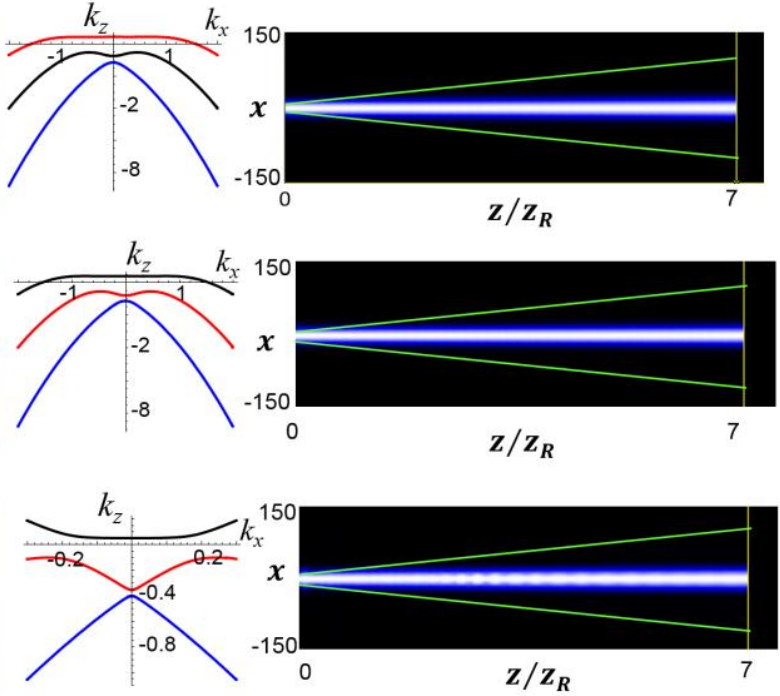

Fig. 1. Self-collimation in $2 \mathrm{D}$ complex crystals. The first/second rows display a schematic representation of the real/imaginary modulations of the optical potential, and the third merges both modulations where the inset indicates the coupling provided by the reciprocal lattice vectors - symmetric in (a), (b) and asymmetric in (c)-. The forth row depicts the real part of dispersion for the specific geometries and modulations amplitudes supporting self-collimation and the firth shows the corresponding numerical simulation of non-diffractive light beam propagation within the corresponding crystal: $(\mathrm{a}, \mathrm{b}) \mathrm{P}$ symmetric 2D lattices for $\varphi=0$ and $\varphi=\pi / 4$ (c) PT-symmetric lattice in the transverse, $x$, direction. The green lines in the fifth row are displayed for comparison to propagation of the same beam in an equivalent homogeneous medium.

\section{CONCLUSIONS}


We show that 2D complex lattices provide an efficient platform to control the dispersive properties of light beams. The simultaneous suppression of diffraction and diffusion is achieved for different complex lattices, and for the phase potential, $\varphi$, ranging from 0 to $\pi / 2$. While PT-symmetric lattices, $\varphi=\pi / 2$, support non-diffractive regimes, either for PhC-like and GLM-like structures, intrinsic diffusion persists in GLM-like case. The predicted self-collimation effect in complex lattices may be also useful to shape the beam profile in integrated optics or optical devices.

\section{ACKNOWLEDGEMENT}

The work is financially supported by Spanish Ministerio de Educación y Ciencia through project FIS201565998-C2-1-P, NATO project SPS-985048 and Erasmus Mundus Doctorate Program Europhotonics (Grant No. 159224-1-2009- 1-FR-ERA MUNDUS-EMJD).

\section{REFERENCES}

[1] R. Zengerle, "Light Propagation in Singly and Doubly Periodic Planar Waveguides," J. Mod. Opt, vol. 34, p. 1589$1617,1987$.

[2] D.Chigrin, S.Enoch, C. Sotomayor Torres, and G.Tayeb, "Light Propagation in Singly and Doubly Periodic Planar Waveguides," Opt. Exp., vol. 11, p. 1203-1211, 2003.

[3] K. Staliunas, and R. Herrero, "Nondiffractive propagation of light in photonic crystals," Phys. Rev. E, vol. 73, p. 016601, 2006.

[4] K. Staliunas, R. Herrero, and R. Vilaseca, "Subdiffraction and spatial filtering due to periodic spatial modulation of the gain-loss profile," Phys. Rev. A, vol. 80, p. 013821, 2009.

[5] M. Botey, R. Herrero and K. Staliunas, "Light in materials with periodic gain-loss modulation on a wavelength scale," Phys. Rev. A, vol. 82, p. 013828, 2010.

[6] K. G. Makris, R. El-Ganainy, and D. N. Christodoulides, and and Z. H. Musslimani, "Beam Dynamics in PT Symmetric Optical Lattices," Phys. Rev. Lett., vol. 100, p. 103904, 2008.

[7] C. E. Ruter, K. G. Makris, R. El-Ganainy, D. N. Christodoulides, M. Segev, and D. Kip, "Observation of parity-time symmetry in optics," Nat. Phys., vol. 6, p. 192-195, 2010.

[8] S. Longhi, "Invisibility in PT-symmetric complex crystals," J. Phys. A., vol. 100, p. 485302, 2011.

[9] R. Herrero, M. Botey, and K. Staliunas, "Nondiffractive-nondiffusive beams in complex crystals," Phys. Rev. A, vol. 89 , p. 063811,2014

[10] W. W. Ahmed, R. Herrero, M. Botey, and K. Staliunas, "Self-collimation in PT-symmetric crystals," Phys. Rev. A, vol. 95, p. 053830, 2017. 